\title{
Acquired resistance to imatinib and secondary KIT exon 13 mutation in gastrointestinal stromal tumour
}

\author{
FRANÇOIS BERTUCCI ${ }^{1,2}$, ANTHONY GONCALVES ${ }^{1,2}$, GENEVIÈVE MONGES ${ }^{2,3}$, ANNE MADROSZYK ${ }^{1}$, \\ JÉROME GUIRAMAND ${ }^{4}$, VINCENT MOUTARDIER ${ }^{4}$, TETSURO NOGUCHI ${ }^{5}$, \\ PATRICE DUBREUIL ${ }^{6}$ and HAGAY SOBOL ${ }^{2,5}$
}

\begin{abstract}
${ }^{1}$ Département d'Oncologie Médicale, Institut Paoli-Calmettes (IPC) and UMR599 Inserm, Institut de Recherches sur le Cancer de Marseille; ${ }^{2}$ Faculté de Médecine, Université de la Méditerranée; Départements de ${ }^{3}$ Anatomopathologie, ${ }^{4}$ Chirurgie et ${ }^{5}$ Onco-Génétique, IPC; ${ }^{6}$ Laboratoire d'Hématopoï̀se Moléculaire et Fonctionnelle, UMR599 Inserm, Marseille, France
\end{abstract}

Received February 6, 2006; Accepted March 27, 2006

\begin{abstract}
Gastrointestinal stromal tumours (GISTs) are the most common mesenchymal tumours of the gastrointestinal tract. Most of them have an activating mutation of KIT or $P D G F R \alpha$ tyrosine-kinase receptors. Imatinib is a selective tyrosine-kinase inhibitor of ABL, KIT and PDGFR, and provides a clinical benefit in about $85 \%$ of patients with advanced GIST. Unfortunately, secondary resistance following initial responses occurs in most of the cases, and molecular mechanisms are poorly understood. We sequenced KIT and $P G D F R \alpha$ exons from one patient with GIST before and after the development of imatinib resistance. We identified, in addition to a primary mutation in exon 9, a secondary mutation in KIT exon 13 (first kinase domain) in the resistant sample. We demonstrate for the first time the feasibility of sequencing such samples removed by non-surgical biopsies during imatinib therapy. Such a approach, far less invasive than surgery and combined with sequencing, will likely help in better tailoring the treatment of advanced GISTs and understanding the mechanisms of resistance and response to imatinib.
\end{abstract}

\section{Introduction}

Gastrointestinal stromal tumours (GISTs) are the most common mesenchymal tumours of the gastrointestinal tract. Approximately $95 \%$ of GISTs express the KIT receptor tyrosine-kinase (stem cell receptor, CD117 antigen). KIT mutations, present in up to $89 \%$ of GISTs, result in a gain-of-function mutation and ligand-independent constitutive activation of the KIT tyrosine-kinase (1). Constitutive resulting activation of the

Correspondence to: Professor François Bertucci, Département d'Oncologie Médicale, Institut Paoli-Calmettes, 232 Bd. SteMarguerite, 13009 Marseille, France

E-mail: bertuccif@marseille.fnclcc.fr

Key words: biopsy, GIST, imatinib, KIT mutation, resistance downstream KIT signalling pathways (2) results in increased cell proliferation and resistance to apoptosis. These oncogenic mutations are most frequent in exon 11 (juxtamembrane domain), and less common in exons 9, 13, 14 and 17. Resistant to conventional chemotherapy, GISTs represent an example of tumour with an effective molecularly targeted therapy. Imatinib is a tyrosine-kinase inhibitor that selectively targets BCR-ABL in chronic myeloid leukaemia (CML), and KIT and PDGFR $\alpha$ and $\beta$ in GISTs. Imatinib represents a new paradigm of targeted therapy that has revolutionized the treatment of GISTs, providing a clinical benefit in $\sim 85 \%$ of patients with advanced disease $(3,4)$. The mutational status of KIT has been associated with response to imatinib and survival (1). Unfortunately, secondary resistance, after initial response or stable disease, occurs in most of the cases, and molecular mechanisms are poorly understood.

We report one case of GIST with primary mutation of KIT exon 9, which developed secondary resistance associated with the acquisition of an additional mutation of exon 13 during imatinib therapy. Importantly, the sequenced resistant sample was obtained only by ultrasound-guided fine needle aspiration (US-FNA) biopsy, and not by surgery. Such an approach relatively not very invasive will likely help in better tailoring the treatment of advanced GISTs and understanding the mechanisms of resistance.

\section{Case report}

A 58 year-old woman underwent in April 2002, surgery for a tumour of the left ovary. At laparotomy, a voluminous ovarian cystic lesion was visualised, associated to multiple peritoneal nodules. Left annexectomy revealed benign mucinous tumour of ovary, whereas the resection of peritoneal granulations identified a GIST CD $117^{+}$. Two months later, the disease relapsed with development of peritoneal disease, a pelvic mass and two hepatic metastases. The patient was referred to our institution. A colonic endoscopic US-FNA biopsy of the pelvic lesion confirmed the diagnosis of recurrent GIST (spindle cell morphology with immunostaining positive for CD117, CD34 and PKC ; Fig. 1). Imatinib 400 mg/d was given from July 2002. Within 2 months, the patient experienced a tumour response. 


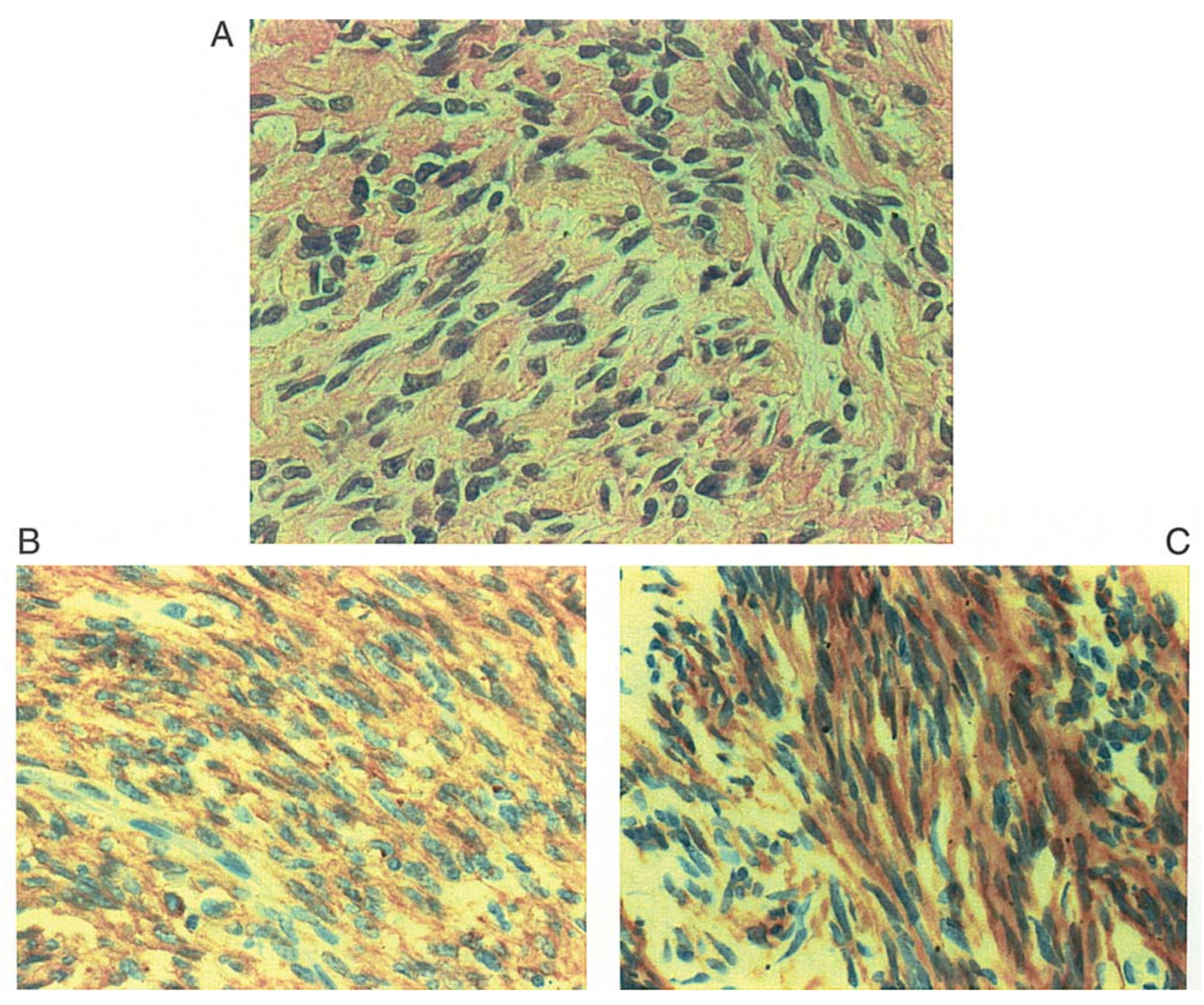

Figure 1. Morphology and immunohistochemistry of the GIST pelvic relapse. (A), Haematoxylin and eosin staining, magnification x20. (B), CD117 immunostaining (clone A4502, 1/50 dilution, Dako). (C), PKC $\theta$ immunostaining. (ref. 610089, 1/50 dilution, Becton-Dickinson).

In September 2003, after 14 months of treatment and a partial response (PR) of pelvi-peritoneal lesions and stabilisation of hepatic lesions, the patient underwent laparotomy. All peritoneal and pelvic lesions were excised. Histology confirmed the diagnosis of ileum GIST with peritoneal extension. After surgery, imatinib was not reintroduced because of patient refusal. Four months later, in January 2004, a progression of hepatic metastases appeared and a non-measurable peritoneal disease. The patient started imatinib $400 \mathrm{mg} / \mathrm{d}$. After 8 weeks of treatment, CT scan showed a PR of the liver lesions and complete regression of the peritoneal relapse that persisted for 12 months. In March 2005, CT imaging revealed strong progression in the size of the hepatic lesions. US-FNA biopsy (18-gauge needle) of a segment IV lesion confirmed the diagnosis and allowed the mutational analysis of a small tumour sample immediately stored in RNA later (Qiagen, Courtaboeuf, France). Imatinib was increased at $800 \mathrm{mg} / \mathrm{d}$, but further progression of hepatic lesions, including that of segment IV, was documented on CT scan after 2 months of treatment. In June 2005, imatinib was stopped and the patient was enrolled in a clinical trial of an investigational agent.

\section{Sequence analysis}

Mutational analyses were done on genomic DNA extracted from two frozen tumour samples. Exons 9-13 and 17 of KIT, and exons 10-21 of PDGFR $\alpha$ were directly sequenced (Fig. 2). They were first amplified by polymerase chain reaction (PCR) using specific primers and HotStarTaq DNA Polymerase (Qiagen). PCR products were purified using MinElute 96 UF (Qiagen), and then sequenced with BigDye Terminator v1.1 Cycle Sequencing kit (Applied Biosystems, Foster City, CA, USA). Cycle sequence products were purified and loaded on an ABI PRISM 3100 Genetic Analyser (Applied Biosystems). Sequencing data were analysed using Sequencing Analysis Software v.5.1 and SeqScape Software v.2.5. The sample from the first peritoneal relapse, sensitive to imatinib and surgically removed after 14 months of treatment, contained a mutation in KIT exon 9 (insertion of 6 bp GCCTAT at nucleotide position 1510, resulting in an insertion Ala-Tyr at codons 502-3). The sample from the imatinib-resistant hepatic metastasis was obtained by non-surgical biopsy; it harboured the same exon 9 mutation associated to an acquired mutation 


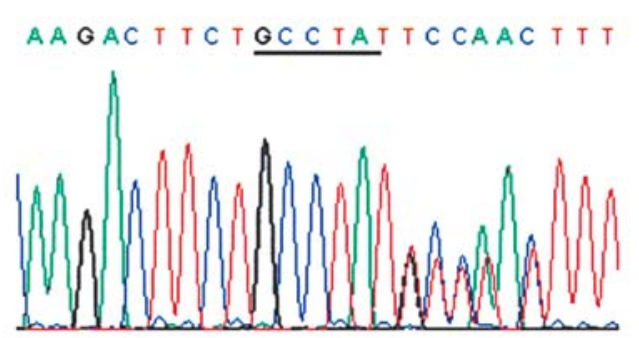

Sensitive sample

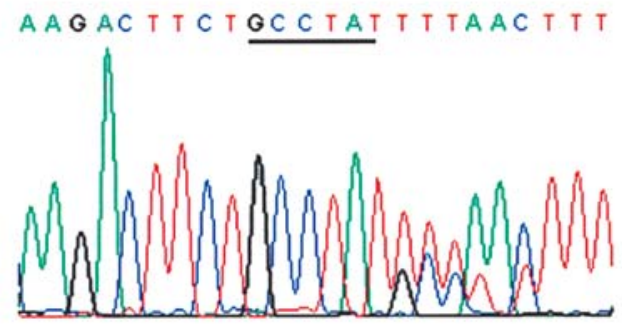

Resistant sample

B

Exon 13

CATGAATATTGTGAATCTACTTGG

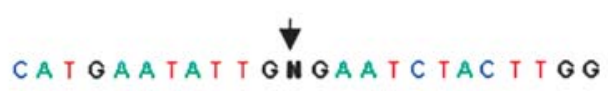

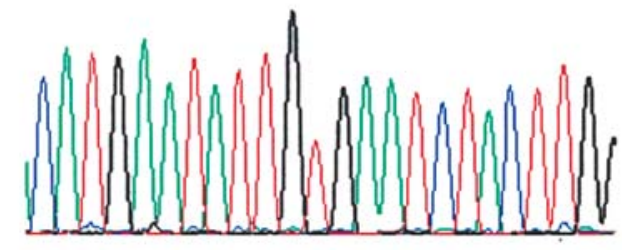

Sensitive sample

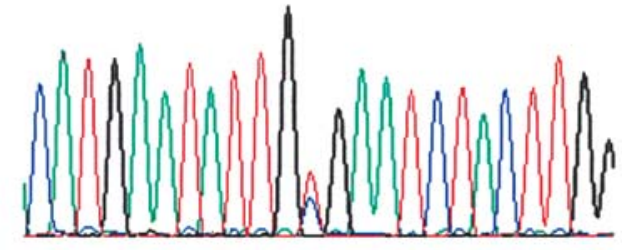

Resistant sample

Figure 2. Sequences of KIT exons 9 and 13 of the sensitive and the resistant samples. The chromatograms of KIT mutations are shown. (A), Genomic sequence of exon 9 showing the same mutation (insertion of 6 bp GCCTAT) in both samples. (B), Genomic sequence of exon 13 showing the wild-type sequence in the sensitive sample and the point mutation $\mathrm{T} \rightarrow \mathrm{C}$ in the resistant sample (indicated by the black arrow).

in $K I T$ exon 13 (point mutation $\mathrm{T} \rightarrow \mathrm{C}$ at nucleotide position 1961, resulting in an amino acid substitution of Val by Ala at codon 654, Val654Ala). This mutation was not present in the imatinib-sensitive tumour sample. The two samples showed wild-type sequence in the other sequenced exons of KIT and PDGFR . Histology and proliferation were similar in the two samples.

\section{Discussion}

Secondary resistance to imatinib is a critical problem in patients with advanced GIST. Our case showed KIT exon 9 mutation in the initial relapse sensitive to imatinib. Such mutation defines a subset of GISTs (10-15\%) that are often located in the small bowel (5), and have a response rate to imatinib of $\sim 48 \%$ and poor clinical outcome (1). In our patient, imatinib yielded initial response of lesions. Secondary hepatic progression occurred under imatinib $400 \mathrm{mg} / \mathrm{d}$ and remained refractory to the dose of $800 \mathrm{mg} / \mathrm{d}$. Sequencing of a resistant hepatic lesion identified additional KIT exon 13 mutation, suggesting a likely cause of acquired resistance. In CML, second mutation in $B C R-A B L$ in critical binding sites for imatinib is the predominant mechanism of acquired resistance $(6,7)$. Similarly, acquired mutations in the EGFR kinase domain have been reported in non-small cell lung cancer patients who develop resistance to gefitinib or erlotinib (8). In GIST, mechanisms of secondary resistance are less well defined. While no more than one activating KIT mutation has been described in untreated GISTs, the identification of a secondary mutation in addition to the activating mutation has been recently reported in GISTs that developed resistance under imatinib, suggesting a causal relationship (9-16). Table I summarizes published cases of secondary resistant GISTs with mutational analyses. Out of the 59 analysed nodules (52 patients), 39 harboured an acquired KIT mutation (31 patients). These mutations have not been reported in untreated GISTs, and are located in the two kinase domains of KIT (exons 13 and 14 for the first domain, and exon 17 for the second one), supporting their association with the development of secondary resistance to imatinib. In all but one case of deletion, they are point mutations. That is in contrast with untreated GISTs, where the incidences of point mutations and of kinase domain mutations are inferior to 15 and $\sim 1 \%$, respectively (17). Concerning exon 13 , the point mutation described in primary GISTs before imatinib therapy is rare and represents a mutation Glu642Lys. The secondary mutation harboured by our patient is even rarer and has been reported in only 13 resistant samples, and results in Val654Ala substitution. Although the exon 17 is the site most frequently concerned by such acquired resistance-associated alterations, this exon 13 mutation is the most common secondary mutation. In most of cases, it is associated to primary mutation of exon 11 . To our 
Table I. Results of sequencing of GIST samples with secondary imatinib resistance reported in literature.

\begin{tabular}{|c|c|c|c|c|}
\hline Refs. & $\begin{array}{l}\text { No. of } \\
\text { patients }\end{array}$ & $\begin{array}{l}\text { Primary } \\
\text { mutation }\end{array}$ & $\begin{array}{l}\text { No. of } \\
\text { sequenced } \\
\text { resistant } \\
\text { samples }\end{array}$ & Secondary mutation \\
\hline (9) & 1 & exon 11 & 1 & exon 14 (Thr670Ile) \\
\hline (10) & 1 & exon 11 & 1 & exon 17 (Tyr823Asp) \\
\hline (11) & 5 & 1 exon 9-4 exon 11 & 6 & 6 exon $13\left(\right.$ Val654Ala) ${ }^{a}$ \\
\hline (12) & 1 & exon 11 & 1 & exon 13 (Val654Ala) \\
\hline (13) & 25 & 6 exon $9-19$ exon 11 & 25 & $\begin{array}{l}4 \text { exon } 13 \text { (Val654Ala) }-3 \text { exon } 14 \text { (Thr670Ile)-5 exon } 17 \\
\text { (Asp716Asn, Asp816Gly, Asp820Glu, Asp820Tyr, Asn822Lys) }\end{array}$ \\
\hline (14) & 2 & 1 exon $9-1$ exon 11 & 7 & $\begin{array}{l}1 \text { exon } 13 \text { (Val654Ala)-2 exon } 14 \text { (Thr670Ile)-4 exon } 17 \\
\text { (Asp816Glu, Asp820Glu, Asn822Lys, Tyr823Asp) }\end{array}$ \\
\hline$(15)$ & 15 & 3 exon $9-11$ exon 11 & 16 & $\begin{array}{l}1 \text { exon } 13 \text { (Val654Ala)-1 exon } 14 \text { (Thr670Ile)-6 exon } 17 \\
\text { (Asp820Tyr, 3; Asn822Lys, 2; Tyr823Asp) }\end{array}$ \\
\hline$(16)$ & 2 & 2 exon 11 & 2 & 2 exon 17 (Asp816His, Arg-Asp815-816del) \\
\hline Present study & 1 & exon 9 & 1 & exon $13(\text { Val654Ala })^{a}$ \\
\hline
\end{tabular}

aThree cases with exon 9 primary mutation and exon 13 secondary mutation.

knowledge, our case represents the third case that combines exon 9 and exon 13 mutations.

Because KIT mutations are considered as a major event in the pathogenesis of GISTs, it is also conceivable that a single missense mutation in kinase domain results in resistance to imatinib. In vitro studies are warranted to confirm the direct role of this exon 13 mutation in resistance to imatinib. Ex vivo the KIT autophosphorylation is not inhibited by high concentrations of imatinib in GIST cells harbouring this mutation, while it is inhibited by the alternative KIT and PDGFR inhibitor PKC412 (13). Although our patient was refractory to $800 \mathrm{mg} / \mathrm{d}$, like other patients with secondary kinase domain mutations, it is unknown whether an initial dose of $800 \mathrm{mg} / \mathrm{d}$ could have prevented the development of resistance. It is also unknown if this mutation is oncogenic alone, but it is likely that it re-activates the activating potential of the primary mutation by inhibiting the binding imatinib-KIT. Based on the crystal structure of the KIT kinase in complex with imatinib (18), the Val654Ala mutation likely eliminates hydrophobic interactions of the methyl groups in the first tyrosine kinase domain with the aromatic rings in imatinib, thereby reducing the binding imatinib-KIT.

Different interpretations, not mutually exclusive, of the presence of such secondary mutation in samples from imatinibtreated patients are possible: either a secondary event favoured by the genetic instability of the allele that harbours an exon 9 mutation (previous reports have shown that the novel exon 13 mutation occurs in the same original mutated allele), either the selection by treatment of a very low level of pre-existing imatinib-resistant clones that have the mutation before treatment but are not detected by lack of sensitivity. More sensitive techniques could address this clinically relevant issue, which might help to predict the risk of secondary resistance under imatinib.

Another important issue raised by this report relates to the mode of removal of the resistant sample in a sequencing objective. All of the reported secondary mutations have been identified on samples surgically removed. In our case, the resistant sample was obtained only by US-FNA biopsy of a liver metastasis and immediate storage in RNA later. With this material, we were able to document both the original exon 9 mutation and the secondary exon 13 mutation. We believe that the reliable sequencing from non-surgical biopsies is an important issue for clinical management of patients with advanced GIST (19), not only in a diagnostic context, but also for helping the monitoring of therapy by sequential biopsies during treatment. Coupled to modern imaging techniques to identify the most metabolically active lesions, such approach far less invasive than surgery offers the opportunity to evaluate in patients with advanced disease and poor clinical status molecular changes during treatment, then to tailor secondline therapy to the underlying mechanism of resistance. With the next availability of drugs alternative to imatinib (SU11248, PKC412 for example) and because different mutations confer differential sensitivity to these drugs, re-biopsy of progressive sites of disease might become a standard procedure and their mutational status should affect the therapeutic choice (surgery, new drugs) after first-line imatinib. Although its clinical utility remains to be proven, we believe that US-FNA with molecular 
analyses will greatly facilitate analysis of sensitive as well as resistant GIST samples, boosting the elucidation of imatinib response-resistance mechanisms as well as the development of second generation targeted therapies in GIST (20).

\section{References}

1. Heinrich MC, Corless CL, Demetri GD, et al: Kinase mutations and imatinib response in patients with metastatic gastrointestinal stromal tumor. J Clin Oncol 21: 4342-4349, 2003.

2. Duensing A, Medeiros F, McConarty B, et al: Mechanisms of oncogenic KIT signal transduction in primary gastrointestinal stromal tumors (GISTs). Oncogene 23: 3999-4006, 2004.

3. Joensuu H, Roberts PJ, Sarlomo-Rikala M, et al: Effect of the tyrosine kinase inhibitor STI571 in a patient with a metastatic gastrointestinal stromal tumor. N Engl J Med 344: 1052-1056, 2001.

4. Demetri GD, von Mehren M, Blanke CD, et al: Efficacy and safety of imatinib mesylate in advanced gastrointestinal stromal tumors. N Engl J Med 347: 472-480, 2002.

5. Antonescu CR, Sommer G, Sarran L, et al: Association of KIT exon 9 mutations with non-gastric primary site and aggressive behavior: KIT mutation analysis and clinical correlates of 120 gastrointestinal stromal tumors. Clin Cancer Res 9: 3329-3337, 2003.

6. Gorre ME, Mohammed M, Ellwood K, Hsu N, Paquette R, Rao PN and Sawyers CL: Clinical resistance to STI-571 cancer therapy caused by BCR-ABL gene mutation or amplification. Science 293: 876-880, 2001

7. Shah NP, Nicoll JM, Nagar B, Gorre ME, Paquette RL, Kuriyan J and Sawyers CL: Multiple BCR-ABL kinase domain mutations confer polyclonal resistance to the tyrosine kinase inhibitor imatinib (STI571) in chronic phase and blast crisis chronic myeloid leukemia. Cancer Cell 2: 117-125, 2002.

8. Pao W, Miller VA, Politi KA, et al: Acquired resistance of lung adenocarcinomas to gefitinib or erlotinib is associated with a second mutation in the EGFR kinase domain. PLoS Med 2: e73, 2005.

9. Tamborini E, Bonadiman L, Greco A, et al: A new mutation in the KIT ATP pocket causes acquired resistance to imatinib in a gastrointestinal stromal tumor patient. Gastroenterology 127 : 294-299, 2004.
10. Wakai T, Kanda T, Hirota S, Ohashi A, Shirai Y and Hatakeyama K: Late resistance to imatinib therapy in a metastatic gastrointestinal stromal tumour is associated with a second KIT mutation. Br J Cancer 90: 2059-2061, 2004.

11. Chen LL, Trent JC, Wu EF, et al: A missense mutation in KIT kinase domain 1 correlates with imatinib resistance in gastrointestinal stromal tumors. Cancer Res 64: 5913-5919, 2004.

12. Tamborini E, Gabanti E, Lagonigro MS, Negri T, Pilotti S, Pierotti MA and Pricl S: KIT/Val654 Ala receptor detected in one imatinib-resistant GIST patient. Cancer Res 65: 1115, 2005.

13. Debiec-Rychter M, Cools J, Dumez H, et al: Mechanisms of resistance to imatinib mesylate in gastrointestinal stromal tumors and activity of the PKC412 inhibitor against imatinib-resistant mutants. Gastroenterology 128: 270-279, 2005.

14. Wardelmann E, Thomas N, Merkelbach-Bruse S, et al: Acquired resistance to imatinib in gastrointestinal stromal tumours caused by multiple KIT mutations. Lancet Oncol 6: 249-251, 2005.

15. Antonescu CR, Besmer P, Guo T, et al: Acquired resistance to imatinib in gastrointestinal stromal tumor occurs through secondary gene mutation. Clin Cancer Res 11: 4182-4190, 2005.

16. Grimpen F, Yip D, McArthur G, et al: Resistance to imatinib, low-grade FDG-avidity on PET, and acquired KIT exon 17 mutation in gastrointestinal stromal tumour. Lancet Oncol 6: 724-727, 2005.

17. Lux ML, Rubin BP, Biase TL, et al: KIT extracellular and kinase domain mutations in gastrointestinal stromal tumors. Am J Pathol 156: 791-795, 2000.

18. Mol CD, Dougan DR, Schneider TR, et al: Structural basis for the autoinhibition and STI-571 inhibition of c-Kit tyrosine kinase. J Biol Chem 279: 31655-31663, 2004.

19. Demetri GD, Titton RL, Ryan DP and Fletcher CD: Case records of the Massachusetts General Hospital. Weekly clinicopathological exercises. Case 32-2004. A 68-year-old man with a large retroperitoneal mass. N Engl J Med 351: 1779-1787, 2004 .

20. Borden EC, Baker LH, Bell RS, et al: Soft tissue sarcomas of adults: state of the translational science. Clin Cancer Res 9: 1941-1956, 2003. 